\title{
Investigation of Stator Wake Variability in Single Stage Axial-Compressor
}

\author{
Xiaorong Xiang, Bo Liu, Liqing Qiao, Qingwei Wang \\ School of Power and Energy, Northwestern Polytechnical University, Xi' an, China \\ E-mail:xiangxiaorong@mail.nwpu.edu.cn \\ Received April 22, 2010; revised June 22, 2010; accepted June 22, 2010
}

\begin{abstract}
In this paper, we investigate the stator wake variability along the axial direction under different rotating speeds and mass flow conditions, in the terms of wake-core position, wake depth, wake width and wake velocity gradient. Experimental study is carried out in a single stage axial compressor by using Hot-wire Anemometer (HWA). Simultaneously, the numerical calculation is brought out in the same experiment rig. Firstly, we analyze the time-averaged velocity and radial vorticity at different axial positions, and explore the wake variability along the axial direction. Then, we present the effects of operation conditions on the wake-core position, and find out the inlet Mach number and incident angle are both the key influence factors of the wake-core position. Finally, we summarize four preliminary conclusions of the wake variability.
\end{abstract}

Keywords: Axial-Flow, Compressor, Wake, Hot-Wire Anemometer (HWA), Vorticity

\section{Introduction}

In the turbo-machine, when the boundary layers, on the blade suction and pressure surface, meet at the trail edge, the mixing area with total pressure and velocity loss forms the wake region. In the multistage turbo-machine, the downstream rows will be influenced by the wake of the upstream rows and appear unsteady effect, then it causes to the aero-dynamical and flow characteristic variation, and plays an important role in the flow loss of the turbo-machine [1-3]. So, it is very significative to bring out further research of the wake flow characteristic in the turbo-machine, for the further purpose to study the unsteady effect, control the flow loss, and improve efficiency of the turbo-machine. Presently, abundant investigations of the wake flow mainly focus on the generation and development of the wake vortex structure [4-6], also on the unsteady effect of the wake flow to the downstream rows [7-9]. But, few research works have been brought out on the influence factors of the wake configuration. Nicole L. Key [10] etc have brought out some experimental studies of the variation orderliness of the rotor wake in a three stages axial-compressor, especially analyzed the influence of the rows interaction on the rotor wake flow under the designed and high-load operation conditions. S. T. Hsu [11] etc have measured the rotor wake flow of a single stage axial-compressor including inlet guide vane, and studied the variation of the wake configuration along with the adjustment of the axial gap between the rotor and stator rows, under the designed and high-load operation conditions respectively. The above two research works all indicate that the variation of the operation conditions and rows interaction play a very important role of the wake configuration transformation. So that it is very important and necessary in the theory and engineering field, to bring out further investigation of the statistic aero-kinematic parameters variation of the axial-compressor wake region under different operation conditions.

In this paper, investigation was carried out on the wake variability along the axial direction under different rotating speeds and mass flow conditions. Experimental study of the stator blade wake in a single stage axial flow compressor was carried out by using Hot-wire Anemometer (HWA). Simultaneously, the numerical research was brought out in the same experiment rig. Experiment was taken at the $7.6 \%, 15.2 \%, 30.3 \%$ and $45.5 \%$ stator chord downstream to the trail edge respectively. The statistic aero-kinematic parameters, including time-averaged velocity and the radial vorticity, were analyzed for the purpose to summarize the wake variability under different operation conditions. The research results can provide experimental and numerical reference for the further investigation of the wake unsteady effect to the downstream rows and flow loss control in the mul- 
tistage axial-compressor under the designed and off-designed operation conditions.

\section{Experimental Equipment and Scheme}

\subsection{Experimental Equipment Introduction}

Figure 1 is a sketch of the experimental rig and the Hot-wire Anemometer (HWA) system. The experimental rig is a single stage axial-compressor including the transparent shroud, and it is driven by a three-phase asynchronous motor. Through the transducer to change the power frequency $(0 \sim 50 \mathrm{~Hz})$, the rotating speed can be adjusted from 0 to $3000 \mathrm{rpm}$ continuously. The compressor mass flow can be adjusted by rotating the throttle at the outlet.

For the HWA test probe is fixed on the top of the shroud, it just can move in the radial and axial direction. In order to measure the velocity downstream to the stator trail edge across one cascade spacing, the stator row in this compressor was designed to be capable rotating across two cascade spacing by adjusting the strut fixed on the stator hub. Table 1 presents the basic parameters of this compressor.

Hot-wire Anemometer (HWA), used in this research work, is the production of the DANTEC Company in Danmark. In the experiment, choosing an X-array probe 55P54 as the test probe, and choosing NI PCI 6040E as the A/D board. The velocity and direction calibration of the dual-sensor probe is carried out the calibration wind-tunnel. According to the numerical simulation results, the stator downstream velocity is about $1 \mathrm{~m} / \mathrm{s}$ to 18 $\mathrm{m} / \mathrm{s}$ under the different operation conditions in this experiment, and the flow stream yaw angles, relating to the axial direction, are all below 40 degree. So, in the calibration choosing the velocity range $0.5 \sim 22 \mathrm{~m} / \mathrm{s}$, and the yaw angle range $-40^{\circ} \sim 40^{\circ}$. The calibration curves just as shown in the Figure 2 and Figure 3.

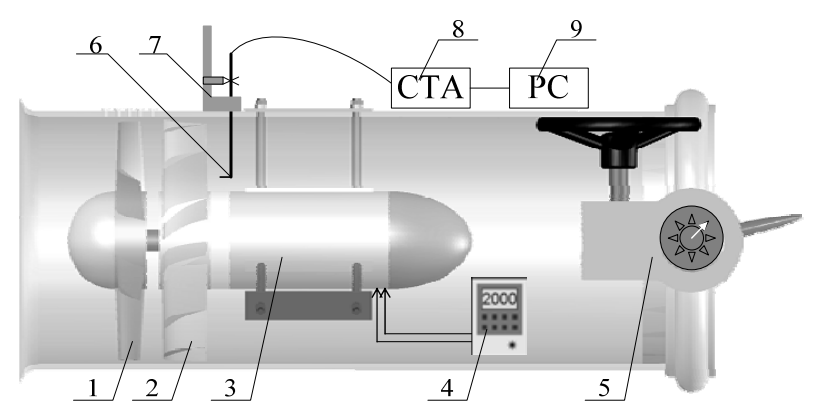

1-Rotor; 2-Stator; 3-Electromotor; 4-Transducer; 5-Throttle; 6-Hot wire probe; 7-Coordinate Frame; 8-CTA; 9-Computer

Figure 1. Sketch of axial-compressor and HWA system.
Table 1. Basic parameter of axial-compressor.

\begin{tabular}{ccccc}
\hline $\begin{array}{c}\text { Rotating } \\
\text { Speed }\end{array}$ & $\begin{array}{c}\text { Shroud } \\
\text { Diameter }\end{array}$ & $\begin{array}{c}\text { Rotor Tip } \\
\text { Gap }\end{array}$ & $\begin{array}{c}\text { Rotor Blade } \\
\text { Number }\end{array}$ & $\begin{array}{c}\text { Rotor Hub } \\
\text { Ratio }\end{array}$ \\
\hline r/min & mm & $\mathrm{mm}$ & & \\
$\mathbf{0 ~ 3 0 0 0}$ & $\mathbf{3 8 3}$ & $\mathbf{7 . 5}$ & $\mathbf{6}$ & $\mathbf{0 . 3 5 1}$ \\
\hline $\begin{array}{c}\text { Stator } \\
\text { Chord }\end{array}$ & $\begin{array}{c}\text { Stator Installa- } \\
\text { tion Angle }\end{array}$ & $\begin{array}{c}\text { Stator Tip } \\
\text { Gap }\end{array}$ & $\begin{array}{c}\text { Stator Blade } \\
\text { Number }\end{array}$ & $\begin{array}{c}\text { Stator Hub } \\
\text { Ratio }\end{array}$ \\
\hline $\mathrm{mm}$ & o(degree) & $\mathrm{mm}$ & & \\
$\mathbf{6 6}$ & $\mathbf{2 2 . 9}$ & $\mathbf{3} \sim \mathbf{5}$ & $\mathbf{1 3}$ & $\mathbf{0 . 3 5 1 ~ 0 . 3 7 6}$ \\
\hline
\end{tabular}

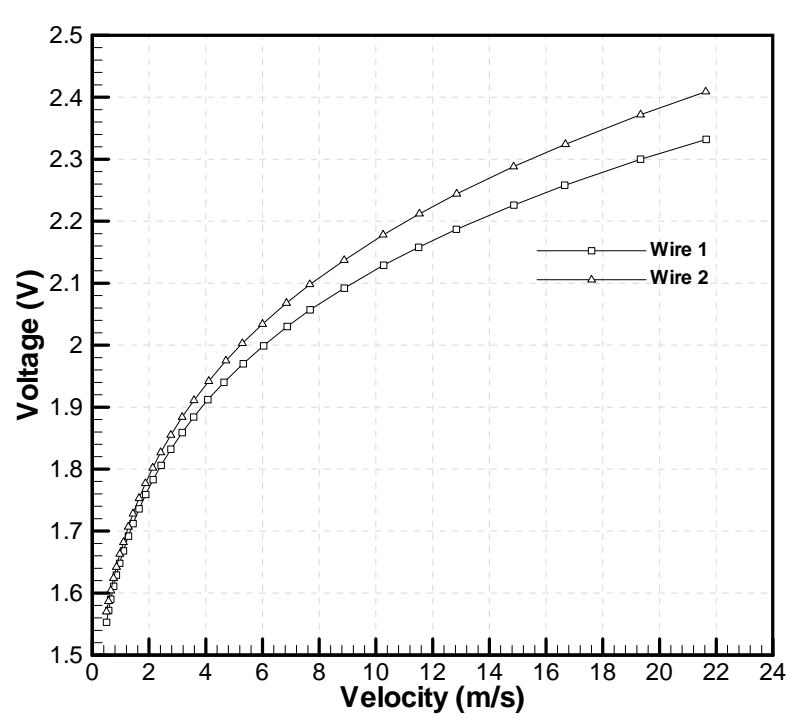

Figure 2. Velocity calibration curve of 55P54 probe.

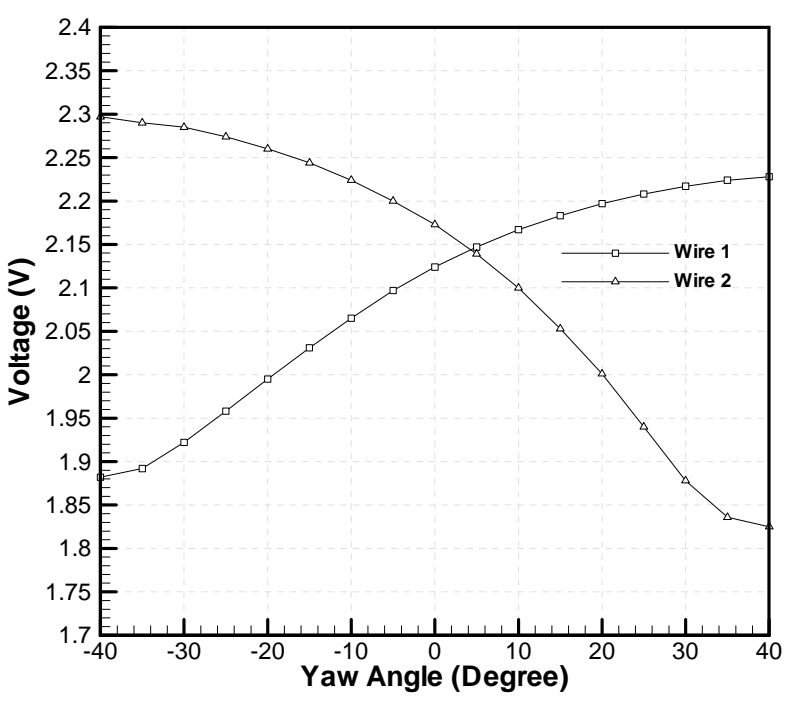

Figure 3. Direction calibration curve of 55P54 probe.

\subsection{Experimental Scheme}

In this research work, the rotating speeds are respectively: 
$n_{1}=1000 \mathrm{r} / \mathrm{min}, n_{2}=1500 \mathrm{r} / \mathrm{min}, n_{3}=2000 \mathrm{r} / \mathrm{min}, n_{4}=$ $2500 \mathrm{r} / \mathrm{min}$, the throttle angles are respectively: $\alpha_{1}=0^{\circ}$, $\alpha_{2}=20^{\circ}, \alpha_{3}=30^{\circ}, \alpha_{4}=40^{\circ}$. The atmosphere conditions are: $p_{0}=0.9771 \times 10^{5} \mathrm{~Pa}, T_{0}=284.05 \mathrm{~K}$.

In order to unify the operation condition of the numerical calculation and experiment, outlet static pressure $p_{2}$ needs to be measured under different rotating speeds and throttle angles, just as shown in Table 2.

Because of the spatial constraint of the experiment rig that the struts are too close to the stator, the test position along the axial direction choosing $z_{1}=7.6 \%, z_{2}=15.2 \%$, $z_{3}=30.3 \%$ and $z_{4}=45.5 \%$ stator chord downstream to the trail edge, at $50 \%$ span-wise section.

The HWA test probe is fixed on the shroud, so the tangential position of the test probe is immovable. In order to measure the velocity across one cascade spacing, it is practicable to rotate the stator in the tangential direction. The tangential measure position is chosen just as shown in the Figures $\mathbf{4}$ and 5, it is 0.5 cascade spacing on both sides of the Stator Blade-2. The cascade spacing in the tangential direction is about $27.7^{\circ}$, and $n(=75)$ measure points is chosen in this direction.

The HWA sampling frequency is $1 \times 10^{4} \mathrm{~Hz}$, sampling time is $1 \mathrm{~s}$. In order to eliminate the sampling stochastic error, the velocity measurement repeats m (=10) times on each test point. After been time averaged, the timeaveraged velocities are averaged by the below formula:

$$
\bar{E}_{k}=\sum_{i=1}^{m} E_{i, k} / m \quad k=1,2, \cdots, n
$$

Table 2. Outlet static pressure under different operation conditions.

\begin{tabular}{ccccc}
\hline$\left(\alpha_{1}=0^{\circ}\right)$ & $1000 \mathrm{rpm}$ & $1500 \mathrm{rpm}$ & $2000 \mathrm{rpm}$ & $2500 \mathrm{rpm}$ \\
\hline$p_{2}(\mathrm{~Pa})$ & $\mathbf{9 7 7 3 4 . 7}$ & $\mathbf{9 7 7 6 2 . 3}$ & $\mathbf{9 7 8 0 0 . 4}$ & $\mathbf{9 7 8 4 9 . 6}$ \\
$(2000$ & $\alpha_{1}=0^{\circ}$ & $\alpha_{2}=20^{\circ}$ & $\alpha_{3}=30^{\circ}$ & $\alpha_{4}=40^{\circ}$ \\
$\mathrm{rpm})$ & $\mathbf{9 7 8 0 0 . 4}$ & $\mathbf{9 7 8 1 2 . 6}$ & $\mathbf{9 7 8 2 6 . 7}$ & $\mathbf{9 7 8 4 2 . 3}$ \\
$p_{2}(\mathrm{~Pa})$ & $\mathbf{9 7 8 2 . 3}$ \\
\hline
\end{tabular}

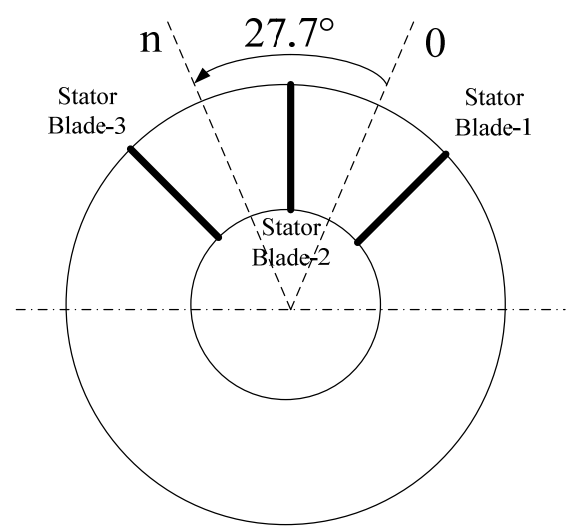

Figure 4. Sketch of tangential measure point in cross section.

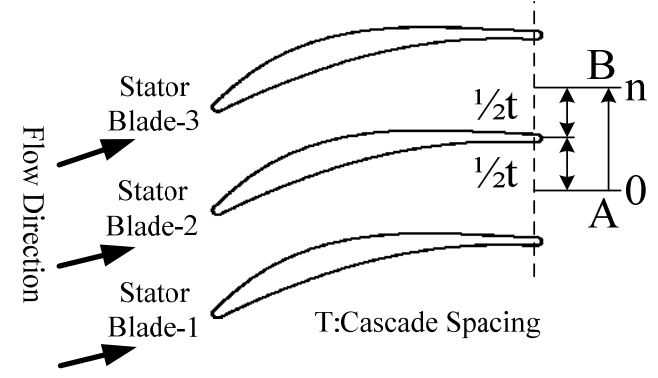

Figure 5. Sketch of stator setting position and tangential measure point in revolution surface.

Under the different operation conditions, the relative standard variances of the time-averaged velocities at different test point are almost less than 5\%. In order to eliminate the high-frequency electromagnetic signal coming from the motor and the transducer, we connect the motor and the transducer to the ground.

\section{Numerical Simulation Method}

The three-dimensional numerical simulation was carried out through applying the NUMECA FINE/TURBO commercial software package, in which the Favre-Reynolds averaged Navier-Stokes equations were discretized by using a cell-center explicit finite volume scheme according to Jameson et al. [12]. And the single equation turbulence model Spalart-Allmaras was chosen to estimate the eddy viscosity. The structural grids, used for the numerical simulation, contained three different topologies as shown in Figure 6. The body-fitted C-type grid topology was used for all the blades, while the H-type grid topology for the main flow region. The butterfly grid topology was chosen for the blade tip gap, the inlet and outlet fairing. In order to consider the influence of the struts at the stator downstream, the struts were dealt with as the stator blades, and calculated together with the rotor and stator upstream. The outlet static pressure was set according to the value in Table 2 under different rotating speeds and throttle angles.

The total grid nodes number of this single axial-compressor, including the struts, were approximately 1700000.

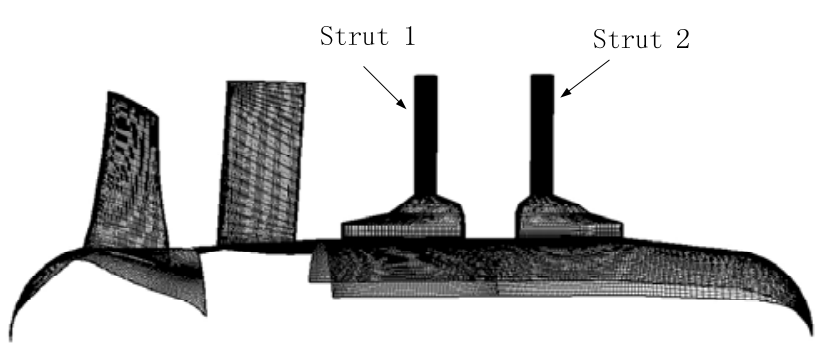

Figure 6. The surface grids of the axial-compressor. 


\section{Experimental and Numerical Results}

In this section, analysis was brought out on the variation of the wake-core position, wake depth, wake width and wake velocity gradient under the different rotating speeds and mass flow conditions. Just in order to summarize the stator wake variability under the different operation conditions.

\subsection{Wake Variability along Axial Direction}

In this subsection, all the results are under $n_{1}$ rotating speed, $\alpha_{1}$ throttle angle, and on the $50 \%$ span-wise section.

Figure 7 gives out the time-averaged axial and tangential velocity in the wake region at $z_{1}$ axial position. The solid line represents the numerical simulation results, while the square point represents the test results. From this figure, we can find out that the numerical and experimental results show the similar variation orderliness: the velocity is nearly unchanged in the free stream region, while appears decrease in the wake region, and the velocity on the wake pressure side reduces more quickly than the wake suction side. The experimental axial velocity value is a little bigger than the numerical result. The tested tangential velocity is very close to the numerical result outside the wake region, while the tested wake depth is larger than the numerical result.

From Figure 8, we can find out that the wake-core position moves to the suction side gradually along the axial direction, the wake becomes much flatter along with it becomes much wider. The time-averaged velocity, outside the wake region, is nearly unchanged.

For the purpose to study the wake-core position and

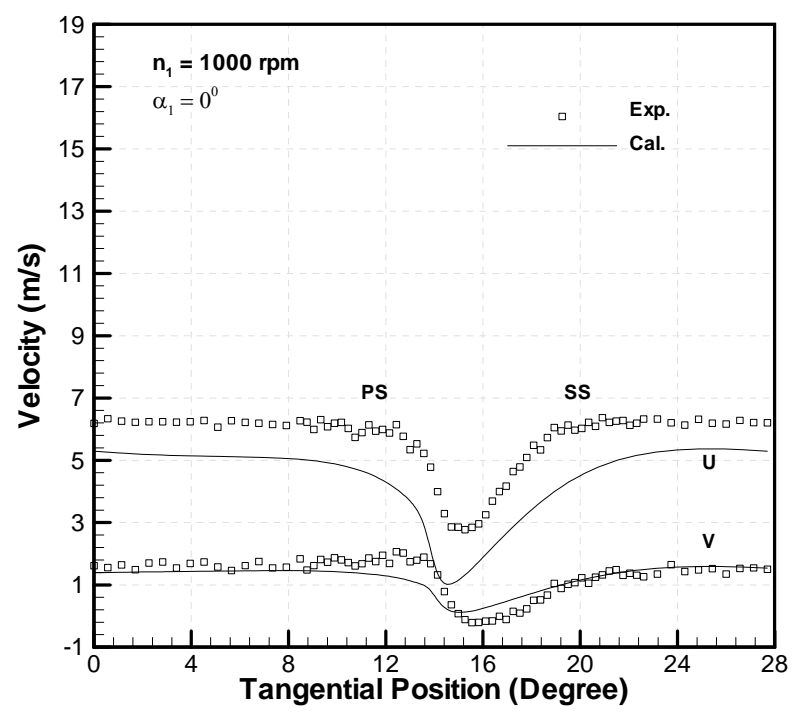

Figure 7. Comparison of mean velocity between the experimental and numerical results.

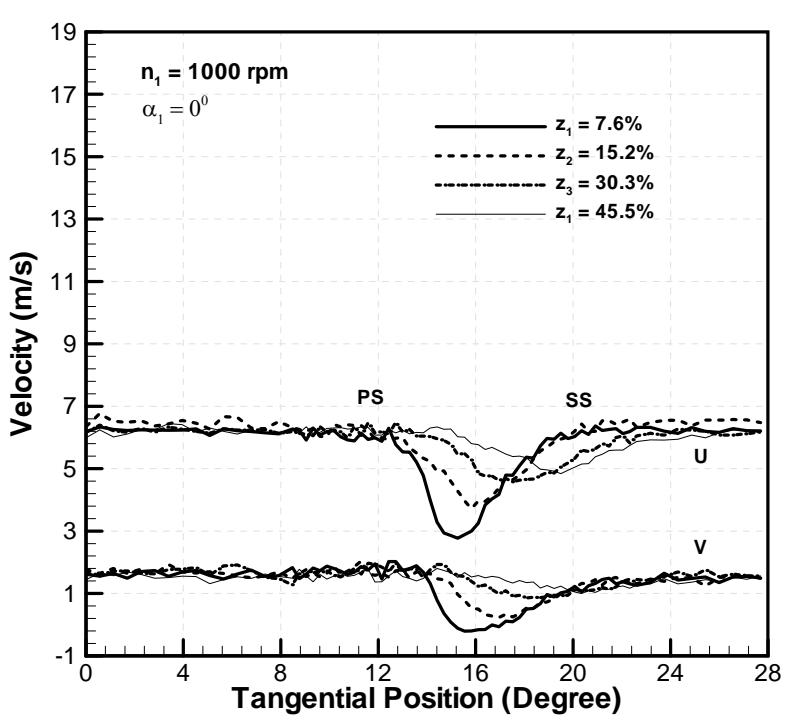

Figure 8. Variation of mean velocity at different axial positions (Experimental results).

depth variability more intuitionistic, it is necessary to bring out the variation curve of the wake-core position and velocity along the axial direction, just as shown in Figure 9. For the experimental results, since the spatial limit of the experiment rip, the shortest distance to the stator trail edge is restricted to $7.6 \%$ chord. For numerical results, because of the position limit of the rotor/stator interface between the stator row and the Strut 1 , the longest distance is restricted to $30.3 \%$ chord. From this figure, we can find out that the gaps between the tested and numerical results in the terms of the wakecore position and wake-core tangential velocity are quite small. Exceptionally, the tested wake-core axial velocity

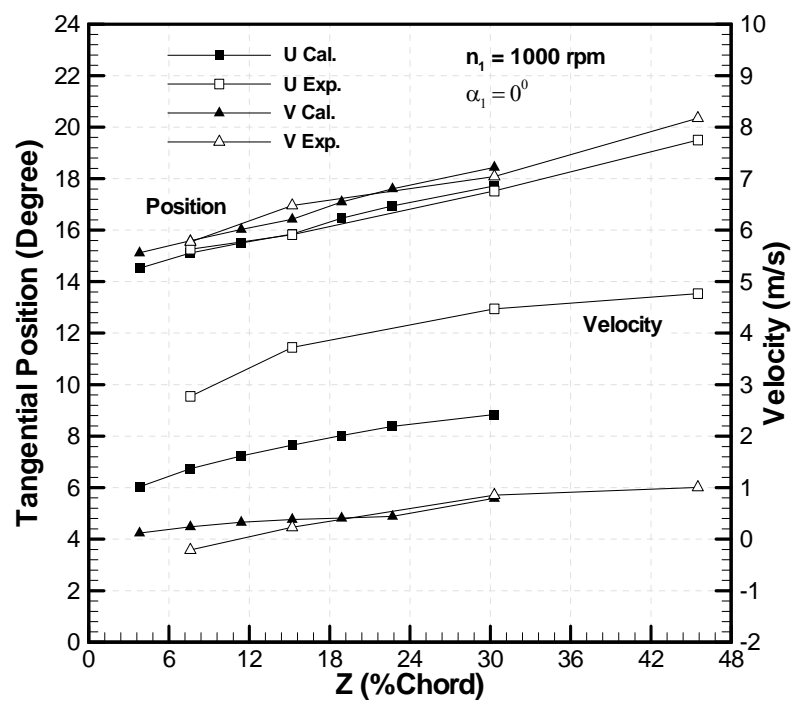

Figure 9. Variation of wake-core position and velocity at different axial positions. 
is bigger than the numerical one. From the variation curve of the wake-core position, it can be found that the wake-core moves to the wake suction side gradually along the axial direction, and the variation curve is nearly linear.

In this paper, we consider the link of the wake-core along the axial direction as the Wake-core Line, and consider the yaw angle between the wake-core line and the axial direction as the Wake-core Yaw Angle. From the Figure 9, it can also be found that the Wake-core Line of the axial velocity is always below the tangential velocity one, but the Wake-core Yaw Angles are almost the same. Since the velocity outside the wake region is nearly unchanged at different axial positions, the high wake-core velocity represents to the small wake depth. The variation curve of the wake-core velocity reflects that the wake depth decreases gradually along the axial direction, because of the influence of the mix of the main flow and the wake, and the decrement of the axial wake depth is larger than the tangential one. Furthermore, the gradient of the wake-core velocity also decreases along the axial direction.

The wake velocity gradient can obviously reflect the position of the velocity inflexion which represents the unsteady point of the wake flow [9], so it is necessary to bring out the analysis of the wake velocity gradient, for the purpose to study the wake variability much deeply.

The radial Vorticity is defined by the below formula:

$$
\xi_{y}=\partial V / \partial z-\partial U / \partial x
$$

In the formula, $\xi_{y}$ represents the radial vorticity, $V$ represents tangential velocity, $U$ represents the axial velocity, $x, y, z$ represents the tangential, radial and axial direction respectively.

For the tangential velocity is nearly unchanged in the main flow region, that is the $\partial V / \partial z$ nearly equals to zero. In the wake region, $\partial V / \partial z$ is also quite smaller compared to the $\partial U / \partial x$. So, the value of the $\xi_{y}$ is mainly influenced by the $\partial U / \partial x$. From the transformation of the radial vorticity, we can approximately summarize the variation orderliness of the velocity gradient in the wake region.

Figure 10 gives out the radial vorticity contours in the wake region. In this figure, the zero vorticity line represents the Wake-core Line of the axial velocity, and its transformation is consistent to the Figure 9. On both sides of the zero line, there are two extremum points at the same axial position, one is positive on the wake pressure side, while the other is negative on the suction side, and the negative one is smaller than the positive one. Along the axial direction, the two extremums reduce gradually, and the positive one decreases much quickly than the negative one. Simultaneously, the extremum points away from the wake core gradually.

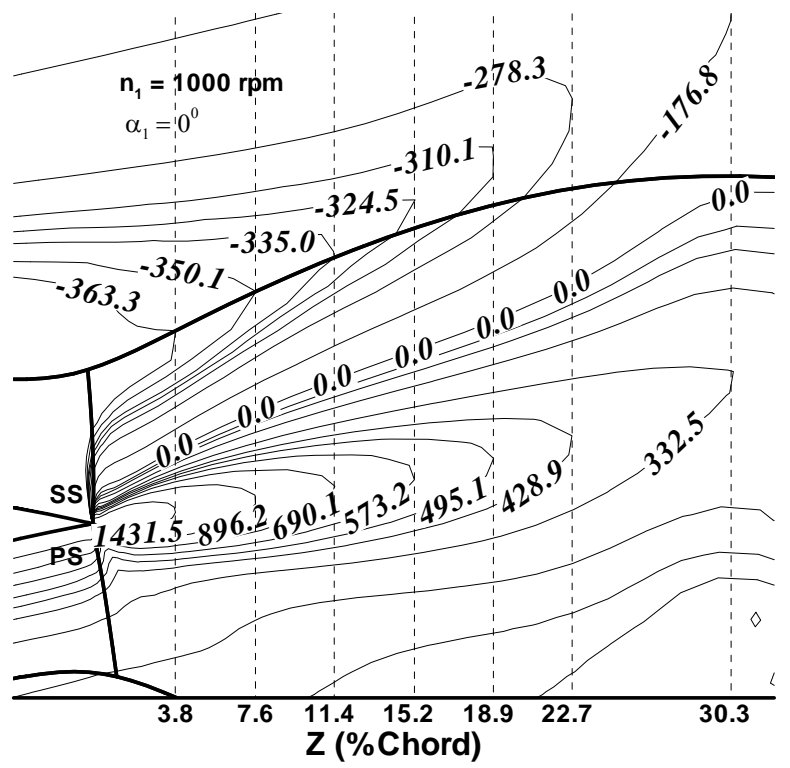

Figure 10. Radial vorticity contours in the stator wake region (Numerical results).

From the above analysis, it is obviously that the vorticity extremum point can approximately represents the axial velocity inflexion, which indicates the unsteady point in the wake region. The high vorticity extremum means the smart variation of the axial velocity in the wake region. From the variation of the vorticity extremum, it can be found that the distance between the velocity inflexion and wake-core increases gradually along the axial direction, on both sides of the wake-core. And the axial velocity on the wake pressure side changes much more acutely compared to the suction side, that is to say, the wake pressure side is more unsteady than the suction side. Along the axial direction, this difference, between the pressure and suction side, becomes more and more inconspicuous, because of the influence of the mixing of the main flow and wake. This variation orderliness is consistent to the experiment results shown in Figure 8.

\subsection{Wake Variability Relating to Operation Condition}

In this subsection, numerical results under different operation conditions will be brought out, for the purpose to analyze the wake variability relating to the operation condition. The variation of the rotating speed and mass flow directly affects the inlet Mach number and the incident angle of the stator blade. So, it is more intuitionistic to summarize the wake variability through analyzing the influence of the inlet Mach number and the incident angle of the stator blade on the wake-core position.

The Figures 11 and 12 respectively give out the variation of the stator inlet flow angle under different rotating 


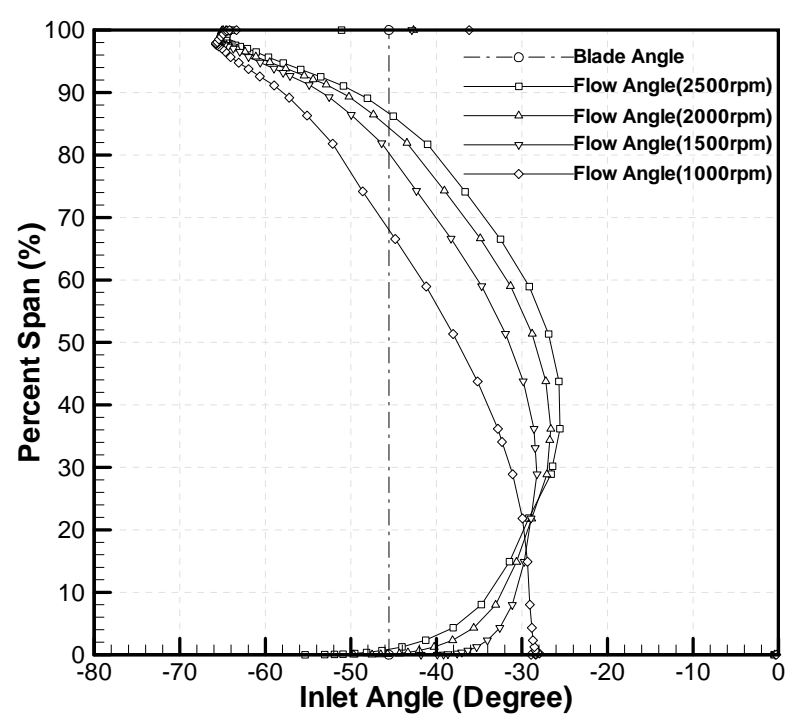

Figure 11. Variation of the stator inlet flow angle under different rotating speeds.

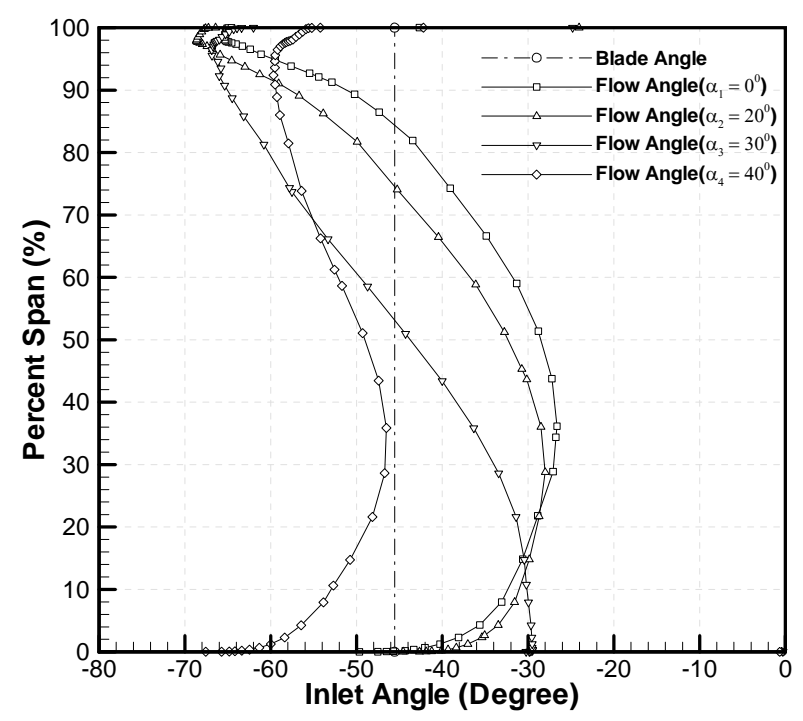

Figure 12. Variation of the stator inlet flow angle under different throttle angles.

speeds and mass flow conditions. The stator blade angle is shown in the both figures (the dash-dot line), so it is convenient to analyze the changes of the incident angle. Just as shown in Figure 11, above the 20\% span-wise section, the incident angle decreases gradually along with the rotating speed increase, while it increase gradually below this section. Above the $15 \%$ span-wise section, the incident angle increases gradually along with the mass flow decrease, while it decrease below this section, just except for the throttle angle $\alpha_{4}$.

Just as shown in the Figure 13, the stator inlet Mach number decreases as the rotating speed reducing. The decrement almost obtains the maximum at the middle

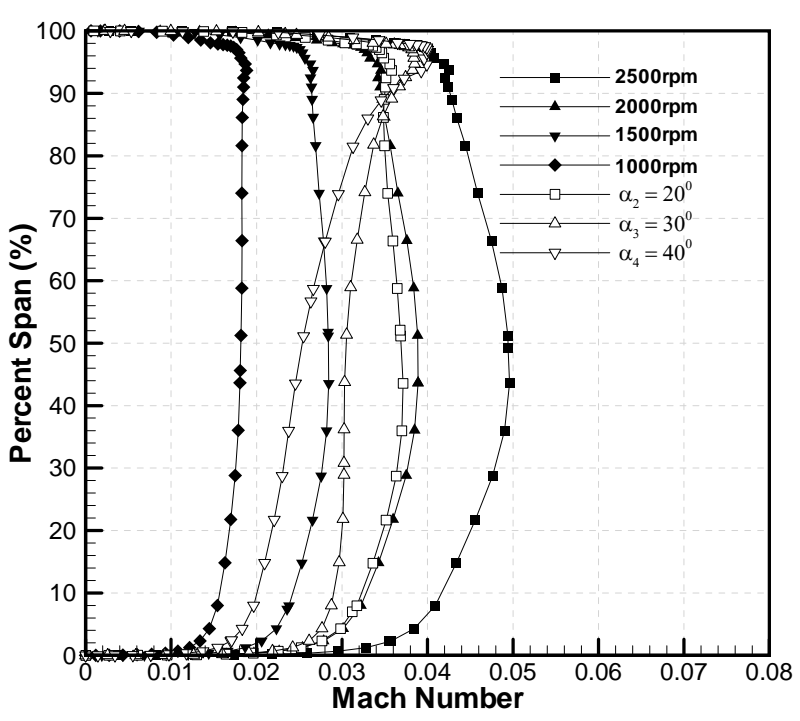

Figure 13. Variation of the stator inlet Mach number under different operation conditions.

span-wise section, and it is nearly symmetrical to the middle section. As the mass flow reducing, the inlet Mach number decreases gradually below the $90 \%$ span-wise section, while it appears increase above this section.

In this part, we will pay attention to the variation of the wake-core position under different operation conditions, just as shown in the Figure 14. For the Wake-core Yaw Angles of the axial and tangential velocity are almost the same, the analysis is carried out just about the axial velocity wake-core in this part.

At $15 \%$ and $20 \%$ span-wise section, the incident angle is nearly unchanged, but the inlet Mach number decreases gradually, respectively as the mass flow and the rotating speed decreasing. At the $20 \%$ section, the wake-core position which nearby the stator trail edge is nearly unchanged, but the Wake-core Yaw Angle increases gradually as the rotating speed decreasing. Along with the mass flow decrease at the $15 \%$ section, the Wake-core Yaw Angle is nearly unchanged. Until the mass flow decreases to a certain value, the Wake-core Line moves up in the tangential direction. That is to say, the inlet Mach number is an influence factor of the wake-core position.

At $90 \%$ span-wise section, as the mass flow decreasing, the inlet Mach number is nearly unchanged, but the incident angle increases gradually. Exceptionally for the throttle angle $\alpha_{4}$, the incident angle appears decrease comparing to the $\alpha_{3}$ situation. At this section, the Wake-core Yaw Angle increases gradually along the axial direction, and the Wake-core Line moves up in the tangential direction. Especially for the $\alpha_{4}$ situation, the Wake-core Line moves down in the tangential direction, for the incident angle decreases compare to $\alpha_{3}$. That is to 


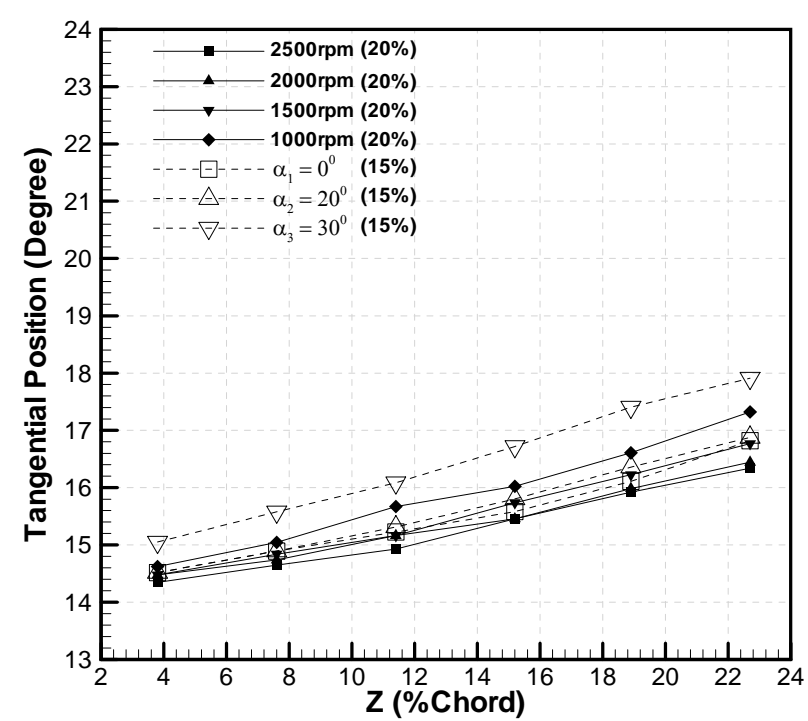

(a)

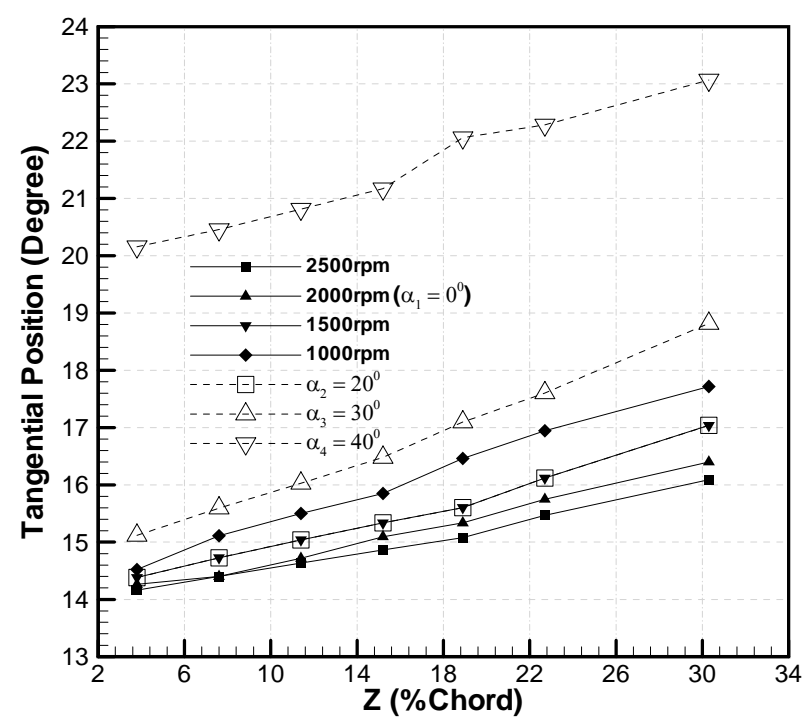

(c)

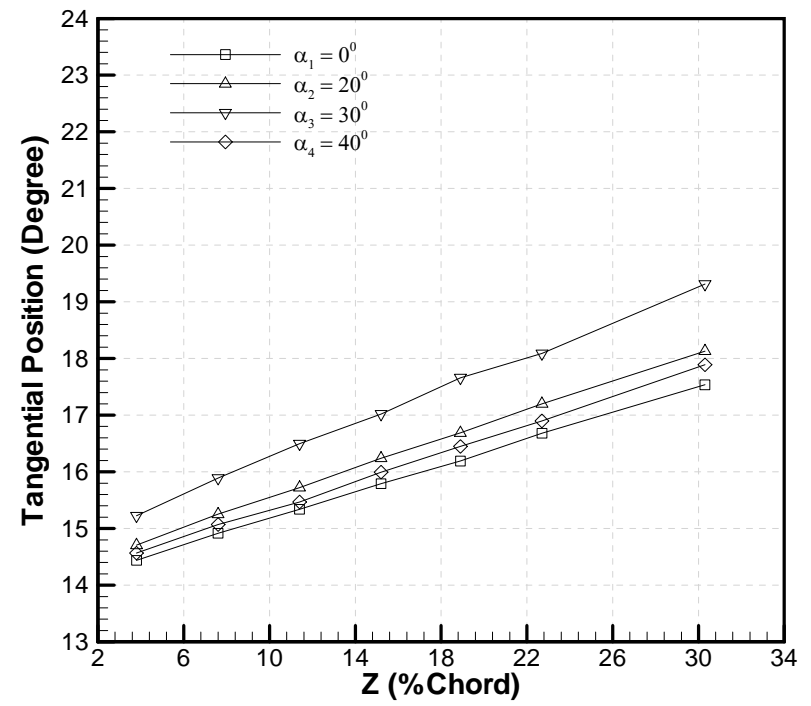

(b)

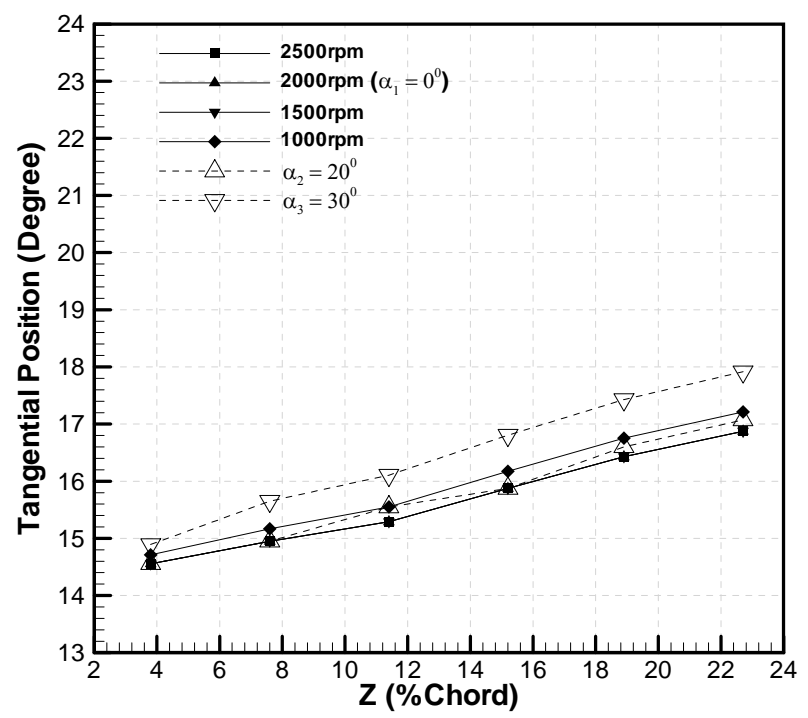

(d)

Figure 14. Variation of wake-core position and velocity under different operation conditions. (a) At $15 \%$ and $20 \%$ span-wise section; (b) At 90\% span-wise section; (c) At 50\% span-wise section; (d) At 10\% span-wise section.

say, the incident angle is another influence factor of the wake-core position, and its influence is opposite to the inlet Mach number.

At 50\% span-wise section, as the rotating speed and mass flow decreasing, the inlet Mach number decreases and the incident angle increases gradually. At this section, the variation of the Wake-core Yaw Angle is similar to the $20 \%$ span-wise section, but the increment is much huger than it. However, at $10 \%$ span-wise section, the variation of the incident angle is opposite to the $50 \%$ section along with the rotating speed and mass flow decrease. At this section, the increment of the Wake-core Yaw Angle is quite smaller than the 50\% section, and also smaller than the $20 \%$ section. There is a special situation, at the $50 \%$ section, when the throttle angle increases from $\alpha_{3}$ to $\alpha_{4}$, the increment of the incident angle is smaller than $\alpha_{2}$ to $\alpha_{3}$, but the wake-core moves up in a huge quantum. This situation can be considered as the flow separation on the suction side.

\section{Conclusions}

Based on the analysis of the time-averaged velocity, the radial vorticity along the axial direction, and the wake-core development under different operation conditions, the following conclusions were drawn:

1) The experimental and numerical results almost 
show the same variation orderliness, the test values are a little bigger than the numerical results.

2) Along the axial direction, the wake-core moves to the wake suction side gradually, and the Wake-core Line of the axial velocity is below the tangential one. The wake width increases gradually along with wake depth decreasing, and the decrement of the wake depth reduces by degrees.

3) The distance between the axial velocity inflexion and the wake-core increases gradually along the axial direction. The axial velocity on the wake pressure side changes more acutely compared to the suction side, and this different reduces by degrees along the axial direction.

4) The inlet Mach number and the incident angle are both the key influence factors of the wake-core position. As the inlet Mach number decreasing and the incident angle increasing, the Wake-core Yaw Angle increases gradually. The wake-core nearby the trail edge is nearly unchanged, until the inlet Mach number (the incident angle) reduces (increases) to a certain value.

\section{References}

[1] D. Gurak, Mehmet S. Kavsaoglu and I. S. Akmandor, "Multi Block Unsteady Navier Stokes Solution of Compressor Stator in Rotor Wake," 43rd American Institute of Aeronautics and Astronautics (AIAA) aerospace sciences meeting and exhibit, Reno, Nevada, 10-13 January 2005, p. 1011.

[2] Albert J. Sanders and Fleeter Sanford, "Rotor Blade-toBlade Wake Variability and Effect on Downstream Vane Response,” Journal of propulsion and power, 2002, Vol. 18, No.2, pp. 456-464.

[3] G. M. Mcnerney, C. P. Van Dam and D. T. Yen Nakafuji, "Blade-Wake Interaction Noise for Turbines with DownWind Rotors,” Journal of solar energy engineering, Vol.
125, No. 4, 2003, pp. 497-505.

[4] M. Roger, "Analytical Modelling of Wake-Interaction Noise in Centrifugal Compressors with Vaned Diffusers," 10th AIAA/CEAS Aeroacoustics Conference, Manchester, 10-12 May 2004, p. 2994.

[5] A. Ferrecchia, W. N. Dawes and P. C. Dhanasekaran, "Compressor Rotor Wakes and Tone Noise Study," 9th AIAA/CEAS Aeroacoustics Conference and Exhibit, Hilton Head, South Carolina, 12-14 May 2003, p. 3328.

[6] Shaobin Li, Fu Chen and Peigang Yan, "Numerical Investigation of the Effect of Stator Clocking on Blade Boundary Layer Flow in Compressor," Acta Aeronautica Et Astronautica Sinica, Vol. 28, No. z1, 2007, pp. s62-69.

[7] J. Estevadeordal, S. Gogineni and L. Goss, "DPIV Study of Wake-rotor Synchronization in a Transonic Compressor," 31st AIAA Fluid Dynamics Conference and Exhibit, Anaheim, CA, 11-14 June 2001, p. 3095.

[8] Bo Liu, Zhipeng Cao and Yuanhu Cai, "Numerical Simulation of Unsteady Vortex in the Wake of Cascade Flow," Journal of Northwestern Polytechnical University, Vol. 23, No. 5, 2005, pp. 562-566.

[9] Guanghua Wang, Baojie Liu and Tao Liu, “A PIV Study of Near Wake Flow of airfoil Part I-Kinematic Characteristics," Journal of Aerospace Power, Vol. 14, No. 2, 1999, pp. 119- 124.

[10] Nicole L. Key, Patrick B. Lawless and Sanford Fleeter, "Rotor Wake Variability in a Multistage Compressor," 45th AIAA Aerospace Sciences Meeting and Exhibit, Reno, Nevada, 8-11 January 2007, p. 19.

[11] S. T. Hsu and A. M. Wo, "Near-Wake Measurement in a Rotor/Stator Axial Compressor Using Slanted Hot-Wire Technique,” Experiments in Fluids, Vol. 23, No. 5, 1997, pp. 441-444.

[12] A. Jameson, W. Schmit and E. Turkel, "Numerical Solution of the Euler Equations by Finite Volume Methods Using Runge-Kutta Time-Stepping Schemes,” 14th AIAA Fluid and Plasma Dynamics Conference, Palo Alto, CA, 23-25 June 1981, p. 1259. 